J. Gynäkol. Endokrinol. AT 2019 • 29:69-75 https://doi.org/10.1007/s41974-019-0103-y Online publiziert: 8. August 2019

(c) Der/die Autor(en) 2019
Katharina Feil · Anna Lena Zippl · Bettina Toth

Universitätsklinik für Gynäkologische Endokrinologie und Reproduktionsmedizin, Medizinische Universität, Innsbruck, Österreich

\title{
Prämature Ovarialinsuffizienz: ESHRE-Leitlinien und aktuelle Studien
}

\section{Definition}

Von einer prämaturen Ovarialinsuffizienz (POI) spricht man bei einem Verlust der Ovarialfunktion vor dem 40. Lebensjahr [1-3]. Dies geht mit Oligobzw. Amenorrhö und erhöhten Gonadotropinspiegeln einher. Die Begriffe prämature Menopause, Climacterium praecox, primäre Ovarialinsuffizienz oder auch "premature ovarian failure“ (POF) werden oft synonym verwendet. Um eine einheitliche, präzise Nomenklatur zu gewährleisten, wird in der aktuellen ESHRE-Leitlinie $\mathrm{zu}$ diesem Thema die Verwendung des Begriffes POI empfohlen [1]

\section{Prävalenz}

Die Prävalenz wird meist mit $1 \%$ angegeben [1-3]. Dies beruht vor allem auf einer Arbeit von Coulam et al. von 1986 [4] und dürfte die derzeitige Prävalenz von POI, nicht zuletzt aufgrund steigender Überlebensraten nach Karzinomen im Kindesalter, unterschätzen. In einer rezent publizierten Kohortenstudie wurde bei Überlebenden nach Krebserkrankungen in der Kindheit eine Prävalenz der POI von $10,8 \%$ festgestellt [5]. Dementsprechend konnte auch in neueren Arbeiten eine Prävalenz für POI in der Allgemeinbevölkerung von $2 \%$ [6] bis hin zu 3,6\% [7] ermittelt werden.

\section{Symptome}

Das typische Zeichen einer POI ist eine Amenorrhö, aber auch Oligomenorrhöen können hinweisend sein. Die
Symptomatik ist meist gekennzeichnet von menopausalen Beschwerden, wie zum Beispiel Hitzewallungen, Schlafstörungen, Konzentrationsstörungen, Libidoverlust oder auch vulvovaginaler Atrophie mit Dyspareunie. Während diese Symptome bei Patientinnen mit einer idiopathischen POI häufig intermittierend auftreten, sind sie bei einer iatrogenen POI vorwiegend persistierend. Im Gegensatz dazu verläuft eine primäre Ovarialinsuffizienz oft symptomlos. Bei den Beschwerden im Rahmen einer POI handelt es sich typischerweise um eine Östrogenentzugssymptomatik.

\section{Diagnostik}

Um die Diagnose einer POI sicherstellen zu können, sollten eine Oligo- bzw. Amenorrhö für mindestens 4 Monate und erhöhte FSH-Werte vorliegen [1-3]. In der ESHRE-Leitlinie werden zwei FSHBestimmungen $>25 \mathrm{IU} / \mathrm{ml}$ im Abstand von mindestens 4 Wochen empfohlen [1]. Andere Leitlinien hingegen nennen zweimalige FSH-Spiegel $>30 \mathrm{IU} / \mathrm{ml}$ im Abstand von 4 bis 6 Wochen als diagnostisches Kriterium [2, 8]. Dies basiert auf einer Arbeit von Goldenberg et al. Die Gruppe konnte 1973 bei Frauen mit primärer Amenorrhö bei FSH-Werten $>33 \mathrm{IU} / \mathrm{ml}$ in Ovarialbiopsien keine Follikel mehr nachweisen [9]. Bei Frauen mit einer autoimmunen Genese dürften die FSH-Spiegel niedriger liegen [10], dies erklärt die ESHRE-Empfehlung mit einem FSH-Grenzwert von $25 \mathrm{IU} / \mathrm{ml}$.

Sonographische Bestimmungen des antralen Follikelcounts (AFC) sind ebenso wie laparoskopische Beurteilungen der
Ovarien mit Biopsien oder auch AMHBestimmung keine validen Diagnosemarker $[1,2]$.

\section{Ursachen}

\section{Genetische Ursachen}

Circa $10 \%$ aller POI-Patientinnen weisen genetische Auffälligkeiten auf, hierunter sind die X-chromosomalen Störungen bei Weitem am häufigsten [1]. Eine Monosomie des X-Chromosoms (sogenanntes Turner-Syndrom) kann in kompletter Ausprägung oder als Mosaik vorliegen. In Abhängigkeit von der Restaktivität können noch eine gewisse Zeit regelmäßige Zyklen auftreten. Patientinnen mit dem Vollbild des Turner-Syndroms haben häufig eine primäre Amenorrhö mit verzögerter bzw. fehlender Pubertätsentwicklung. Bei Vorliegen eines Mosaiks kommt es häufig zu einer spontanen Thelarche und immerhin circa $6 \%$ der Patientinnen haben einen regelmäßigen Zyklus [11]. Bei Mosaiken mit Y-chromosomalen Anteilen besteht ein erhöhtes Risiko für das Auftreten von Gonadoblastomen, daher wird in diesen Fällen eine Gonadektomie empfohlen [12]. Eine Chromosomenanalyse sollte daher Teil der Routineabklärung bei POI sein [1].

Eine weitere $\mathrm{X}$-chromosomale Erkrankung ist das fragile X-Syndrom. Hierbei handelt es sich um eine FMR1Genmutation, die durch eine Verlängerung des CGG-Tripletts verursacht wird. Die Vollmutation mit $>200$ Repeats ist eine der häufigsten Ursachen für eine geistige Behinderung [13]. Die Prämutation mit circa 60-200 Repeats führt 


\begin{tabular}{|c|c|}
\hline \multirow[t]{2}{*}{$\begin{array}{l}\text { Hohes Risiko } \\
\text { (>80\%iges Risiko für eine } \\
\text { POI) }\end{array}$} & $\begin{array}{l}\text { Konditionierung für Stammzelltransplantation (insbeson- } \\
\text { dere alkylanzienbasierte myeloablative Konditionierung } \\
\text { mit Busulfan, Cyclophosphamid, Melphalan) }\end{array}$ \\
\hline & BEACOPP $\times 6-8$ bei Frauen $>35$ Jahre \\
\hline \multirow{5}{*}{$\begin{array}{l}\text { Intermediäres Risiko } \\
\text { (40-60\%iges Risiko für eine } \\
\text { POI) }\end{array}$} & CMF, CEF, CAF, TAC $\times 6$ bei Frauen 30-39 Jahre \\
\hline & AC oder $\mathrm{EC} \times 4 \rightarrow$ Taxan \\
\hline & BEACOPP $\times 6-8$ bei Frauen $25-35$ Jahre \\
\hline & $\mathrm{CHOP} \times 6$ bei Frauen $\geq 35$ Jahre \\
\hline & Knochen- und weichteilsarkomtypische Therapie \\
\hline \multirow{9}{*}{$\begin{array}{l}\text { Niedriges Risiko } \\
\text { (<20\%iges Risiko für eine } \\
\text { POI) }\end{array}$} & CMF, CEF, CAF, TAC $\times 6$ bei Frauen $\leq 30$ Jahre \\
\hline & $A C \times 4$ bei Frauen $\leq 40$ Jahre \\
\hline & BEACOPP $\times 6-8$ bei Frauen $<25$ Jahre \\
\hline & $A B V D \times 2-4$ \\
\hline & $\mathrm{CHOP} \times 6$ bei Frauen $<35$ Jahre \\
\hline & CVP \\
\hline & AML-typische Therapie (Anthrazyklin/Cytarabin) \\
\hline & ALL-typische Therapie („,multi-agent $\left.{ }^{\prime \prime}\right)$ \\
\hline & FOLFOX bei Frauen $\leq 40$ Jahre \\
\hline $\begin{array}{l}\text { Sehr niedriges oder kein } \\
\text { Risiko für eine permanente } \\
\text { Amenorrhö }\end{array}$ & Methotrexat, Fluorouracil, Vincristin \\
\hline \multicolumn{2}{|c|}{$\begin{array}{l}\text { ABVD Doxorubicin, Bleomycin, Vinblastin, Dacarbazin; AC Doxorubicin, Cyclophosphamid; ALL aku- } \\
\text { te lymphatische Leukämie; AML akute myeloplastische Leukämie; BEACOPP Bleomycin, Etopo- } \\
\text { sid, Doxorubicin, Cyclophosphamid, Vincristin, Procarbazin, Prednison; CAF Cyclophosphamid, } \\
\text { Adriamycin, Fluorouracil; CEF Cyclophosphamid, Epirubicin, 5-Fluorouracil; CHOP Cyclophosph- } \\
\text { amid, Doxorubicin, Vincristin, Prednisolon; CMF Cyclophosphamid, Methotrexat, 5-Fluorouracil; } \\
\text { CVP Cyclophosphamid, Vincristin, Prednison; EC Epirubicin, Cyclophosphamid; FOLFOX Folinsäure } \\
\text { (Leucovorin), 5-Fluorouracil, Oxaliplatin; POI prämature Ovarialinsuffizienz; TAC Paclitaxel (Taxol), } \\
\text { Doxorubicin (Adriamycin), Cyclophosphamid }\end{array}$} \\
\hline
\end{tabular}

zu keiner intellektuellen Einschränkung, aber bei $21 \%$ der Betroffenen tritt eine POI auf [14]. Eine genetische Abklärung bei POI sollte auch eine Testung auf eine Prämutation im FMR1-Gen beinhalten [1].

Zahlreiche weitere genetische Erkrankungen können ebenfalls zu einem POI führen. Eine routinemäßige Testung auf autosomale Erbkrankheiten kann jedoch nicht empfohlen werden und sollte auf spezielle Verdachtsfälle beschränkt werden [1].

\section{Autoimmunopathien}

Autoimmunerkrankungen, vor allem Morbus Addison und Immunthyreopathien, sind gehäuft mit POI assoziiert. Im Speziellen trifft dies auf die beiden häufigsten autoimmunen polyglandulären Syndrome (APS), den APS-Typ 1 (Morbus Addison, Hypoparathyreoidismus, Candidiasis) und den APS-Typ 2
TSH-Kontrollen bei positiven Befunden werden bei POI ebenso empfohlen [1].

\section{Infektionen}

Es gibt Fallberichte zum Auftreten einer POI nach Infektionen mit Mumps, HIV, Herpes Zoster, Zytomegalievirus, Tuberkulose, Malaria, Varizellen und Shigellen. $\mathrm{Da}$ es sich hierbei bislang aber vor allem um Einzelberichte handelt, wird ein routinemäßiges Infektionsscreening derzeit nicht empfohlen [1].

\section{latrogen}

Therapeutische Maßnahmen aufgrund einer malignen Erkrankung wie Chemotherapie oder Bestrahlungen im Bereich des kleinen Beckens können ebenso wie operative Maßnahmen an den Ovarien auch bei benignen Erkrankungen (z. B. Endometriose, Ovarzystenausschälung) $\mathrm{zu}$ einer POI führen. Bei Chemotherapeutika sind sowohl das Alter bei Applikation als auch der Wirkstoff und dessen Dosierung von entscheidender Bedeutung für die Gonadotoxizität (• Tab. 1 modifiziert nach [19]). Je älter die Patientin, desto höher das Risiko, in weiterer Folge eine POI zu entwickeln. Eine Strahlenexposition führt ebenfalls dosis- und altersabhängig $\mathrm{zu}$ einem Verlust der Eizellreserve. Bereits 2Gy reduzieren die Follikeldichte um die Hälfte [20]. Bei Neugeborenen wurde eine effektive sterilisierende Strahlendosis von $20,3 \mathrm{~Gy}$, bei Kindern von $18,4 \mathrm{~Gy}$, bei 20 -Jährigen von $16,5 \mathrm{~Gy}$ und bei 30 Jährigen von 14,3 Gy errechnet [21].

Operationen an den Ovarien, im speziellen bilaterale Exzisionen von Endometriomen, können zu einer POI führen [22].

Eine explizite Aufklärung der Patientinnen vor den jeweiligen gonadotoxischen Therapien bezüglich POI ist daher dringend zu empfehlen [1].

\section{Idiopathisch} sitiven Befunden ist in weiterer Folge eine Abklärung bezüglich Morbus Addison durch einen Endokrinologen angezeigt [1]. Eine Bestimmung von Schilddrüsenantikörpern (TPO) und nachfolgende 
schen POI ist unklar, man geht von einem Anteil von 50 bis $90 \%$ aus $[1,23]$.

\section{Kinderwunsch und Fertilität}

Da die Diagnose POI die Patientinnen meist unerwartet in einem normalerweise fertilen Alter trifft, ist ihre Familienplanung häufig noch nicht abgeschlossen. Die Chancen auf eine spontane Schwangerschaft liegen bei 1,5 bis $5 \%$ [24]. Aktuell gibt es keine Therapieansätze, die die Ovulationsrate und damit die Konzeptionschancen zuverlässig steigern. Die Behandlung mit Östradiol, GnRH-Analoga oder Cortison hat keinen signifikanten Anstieg der Ovulationsrate gezeigt [24].

Da POI mit dem Verlust des ovariellen Follikelpools einhergeht, ist eine Fertilitätsprotektion bei manifester POI nicht mehr möglich. Daher ist es von grundlegender Bedeutung, Patientinnen vor einer gonadotoxischen Therapie über die Möglichkeiten der Fertilitätsprotektion aufzuklären. Je nachdem, wie viel Zeit bis zum Therapiebeginn zur Verfügung steht, aber auch je nach vorhandener ovarieller Reserve, Alter und Präferenz der Patientin, stehen verschiedene Methoden zur Verfügung [19].

Am besten etabliert ist die ovarielle Stimulation mit Kryokonservierung von Oozyten oder Embryonen, für die ein Zeitfenster von etwa 2 Wochen benötigt wird.

Alternativ kommt die Kryokonservierung von Ovargewebe infrage. Dabei wird laparoskopisch ein Teil eines Ovars entfernt, aufbereitet und kryokonserviert ([25]; - Abb. 1). Nach der Retransplantation des Gewebes kann eine spontane Konzeption oder eine IVF/ICSI angestrebt werden. Bei dringendem Beginn der Chemotherapie ist ein Vorteil dieser Methode, dass nur sehr wenig Zeit benötigt wird. Bei präpubertären Mädchen ist die Kryokonservierung von Ovargewebe aktuell die einzige Möglichkeit der Fertilitätsprotektion [26].

Bei Erwachsenen besteht eine weitere Möglichkeit der Fertilitätsprotektion in der Verabreichung von GnRH-Analoga. Mehrere Metaanalysen haben in den letzten Jahren eine signifikante Reduktion des POI-Risikos durch GnRHa gezeigt, es ist aber noch unklar, wie lange

J. Gynäkol. Endokrinol. AT 2019·29:69-75 https://doi.org/10.1007/s41974-019-0103-y

(c) Der/die Autor(en) 2019

\section{K. Feil · A. L. Zippl · B. Toth}

\section{Prämature Ovarialinsuffizienz: ESHRE-Leitlinien und aktuelle Studien}

\section{Zusammenfassung}

Der Verlust der Ovarialfunktion vor dem 40. Lebensjahr wird als prämature Ovarialinsuffizienz (POI) bezeichnet und tritt mit einer Prävalenz von 1 bis $2 \%$ auf. Eine POI liegt vor, wenn eine Amenorrhö bzw. Oligomenorrhö seit 4 Monaten besteht und das FSH mindestens zweimalig im Abstand von 4 Wochen $>25 \mathrm{IU} / \mathrm{ml}$ liegt. Genetische Erkrankungen, Autoimmunopathien wie auch gonadotoxische Therapien können ursächlich sein, der wahrscheinlich größte Anteil ist jedoch idiopathisch. Bei einer manifesten POI liegt die spontane Schwangerschaftsrate bei 1,5 bis maximal $5 \%$, meist ist bei Kinderwunsch eine Eizellspende unumgänglich. Vor allem vor einer gonadotoxischen
Therapie sollte daher immer eine Beratung bezüglich Fertilitätsprotektion an einem spezialisierten Zentrum erfolgen. Frauen mit POI haben aufgrund eines erhöhten Risikos für kardiovaskuläre Erkrankungen eine reduzierte Lebenserwartung. Auch eine Osteoporose tritt gehäuft auf, ebenso wie andere Östrogenmangelsymptome. Eine Hormonersatztherapie ist bis zum Eintritt des normalen Menopausenalters indiziert.

\section{Schlüsselwörter}

Prämature Menopause · Climacterium praecox · Amenorrhö · Hormonersatztherapie · Fertilität

\section{Premature Ovarian Failure: ESHRE Guidelines and Current Studies}

\section{Abstract}

The loss of ovarian function before the age of 40 years is called premature ovarian failure (POI) and has a prevalence of $1-2 \%$. In order to be able to diagnose POI, amenorrhea or oligomenorrhea must have been present for 4 months and two FSH measurements $>25 \mathrm{IU} / \mathrm{ml}$ at an interval of 4 weeks. Genetic disorders, autoimmune diseases and gonadotoxic therapies may be the cause, but probably the largest proportion seems to be idiopathic. In a manifest POI, the spontaneous pregnancy rate is 1.5 to a maximum of $5 \%$, usually an egg donation is inevitable. A consultation on fertility protection prior to a gonadotoxic therapy should be offered at a specialized center. Women with POI have a reduced life expectancy due to an increased risk of cardiovascular disease. Osteoporosis also occurs frequently, as well as other estrogen deficiency symptoms. Hormone replacement therapy is indicated until the onset of normal menopause age.

\section{Keywords}

Premature menopause - Climacteric . Amenorrhea $\cdot$ Hormonal replacement therapy · Fertility der Effekt anhält. Zudem gibt es bisher nur unzureichende Daten über die Auswirkung auf die Fertilität. Laut AWMFLeitlinie ist daher die alleinige Gabe von GnRH-Analoga nicht ausreichend [19].

Ist eine Radiatio des Beckens geplant, kann außerdem eine Transposition der Ovarien durchgeführt werden. Die Fertilitätseinschränkung durch die Strahlenschädigung des Uterus kann dadurch aber nicht vermieden werden.

Bei Patientinnen mit Turner-Syndrom und vorhandener ovarieller Funktion kommt eine Fertilitätsprotektion mittels Kryokonservierung von Oozyten,
Embryonen oder Ovargewebe ebenfalls infrage [12, 27].

Hat eine Frau mit bereits manifester POI Kinderwunsch, so ist die Eizellspende die Methode der Wahl. In Österreich ist diese Behandlung seit der Novelle des Fortpflanzungsmedizingesetzes von 2015 erlaubt, allerdings darf die Spenderin maximal 30 Jahre alt sein. Die Kommerzialisierung der Eizellspende ist verboten. Aufgrund dieser Einschränkungen ist die Suche nach einer Spenderin erschwert und für die Patientinnen bleibt häufig nur die Option einer Behandlung im Ausland. 

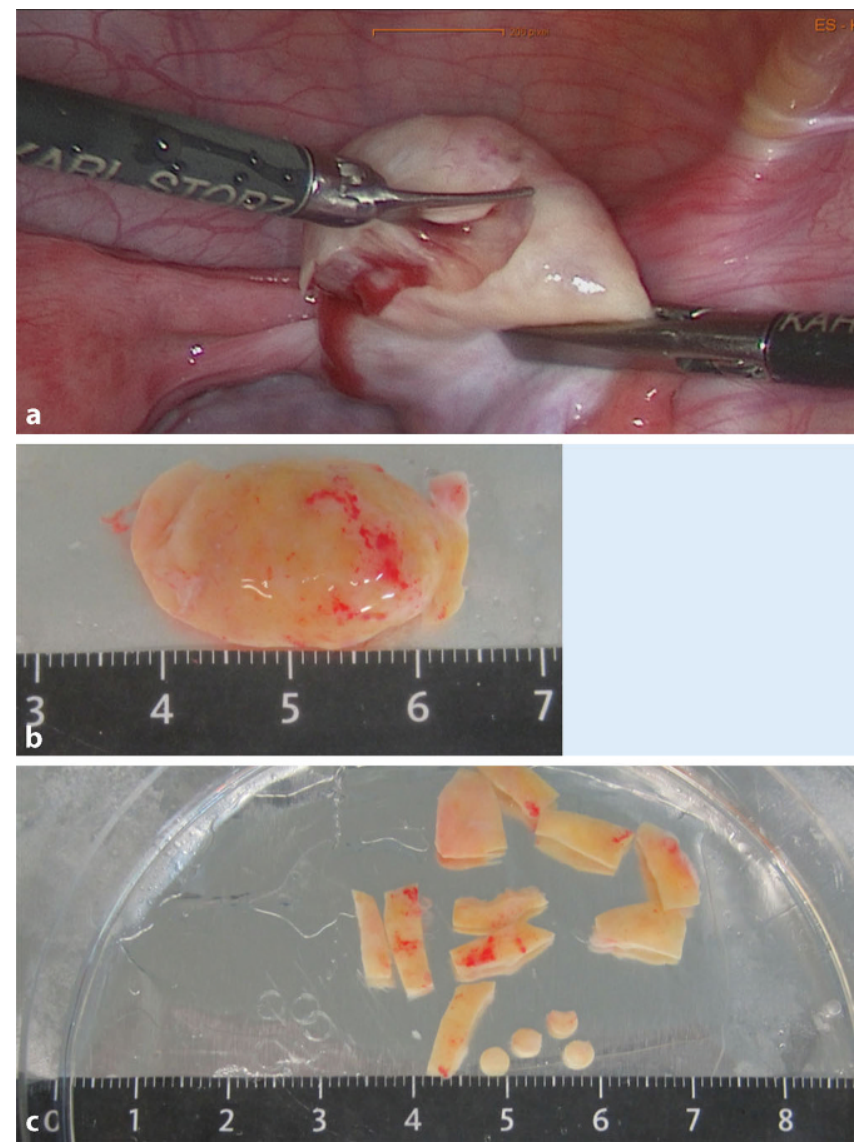

Abb. $1<$ a Laparoskopische Teilresek tion des Ovars vor geplanter Chemotherapie; $\mathbf{b}$ Ovargewebe vor der Verarbeitung; c aufgearbeitetes Ovargewebe vor der Kryokonservierung

\section{POI und Schwangerschaft}

Spontane Schwangerschaften bei Frauen mit idiopathischer POI oder mit POI nach Chemotherapie sind nicht mit höheren geburtshilflichen oder neonatalen Komplikationsraten assoziiert [24, 28]. Eine Ausnahme stellen Behandlungen mit Anthrazyklinen und eine mediastinale Radiotherapie dar [29]. Aufgrund der Kardiotoxizität dieser Therapien sollte in der Schwangerschaft eine kardiologische Mitbetreuung stattfinden. Ist die POI auf eine pelvine Radiotherapie zurückzuführen, kann neben den Ovarien auch der Uterus durch die Strahlung geschädigt sein. Hierbei muss mit Komplikationen wie Spätaborten, Frühgeburten, niedrigem Geburtsgewicht, IUFT, postpartaler Hämorrhagie, Plazentationsstörungen und Uterusruptur gerechnet werden [28]. Schwangere im Z.n. Radiatio des Uterus sollten daher in einem geburtshilflichen Zentrum betreut werden [1].

Ist die Schwangerschaft hingegen durch Eizellspende entstanden, treten geburtshilfliche Komplikationen, vor allem hypertensive Schwangerschaftserkrankungen, Wachstumsretardierung und Frühgeburten, häufiger auf [30]. Daher empfiehlt die ESHRE hier ebenfalls die Betreuung in einem Zentrum.

Schwangerschaften bei Frauen mit Turner-Syndrom sind mit einem hohen Risiko nicht nur für geburtshilfliche, sondern auch für maternale Komplikationen assoziiert. Im Vordergrund stehen kardiovaskuläre Komplikationen [12], sodass neben der Anbindung an ein geburtshilfliches Zentrum auch eine kardiologische Mitbetreuung nötig ist.

Abhängig von der Ursache der POI ist folglich eine gründliche präkonzeptionelle Beratung und ggf. Abklärung auf maternale Risikofaktoren indiziert. In einzelnen Fällen muss von einer Schwangerschaft sogar abgeraten werden.

\section{POI und gesundheitliche Folgen}

Der Östrogenmangel führt unabhängig von der Ursache der POI zu einer Abnahme der Knochendichte und steigert somit das Risiko von Frakturen [31]. Die Prävalenz von Osteoporose liegt bei Frauen mit POI bei $8-14 \%[23,31]$, wobei das Risiko durch ein frühes Auftreten der POI, Verzögerung der Diagnose und Therapie, Bewegungsmangel, niedriges Körpergewicht, Rauchen sowie Vitamin-Dund Kalziummangel gesteigert wird [31]. Die Patientinnen sollten daher zur Vermeidung dieser zusätzlichen Risikofaktoren angehalten werden. Der Dachverband für Osteologie empfiehlt für alle Risikogruppen die Sicherstellung einer Gesamtzufuhr von täglich $1000 \mathrm{mg}$ Kalzium und 800-1000 IE Vitamin $\mathrm{D}_{3}$ durch die Ernährung und/oder Supplemente [32]. Eine Hormonersatztherapie (HRT) auch zur Osteoprotektion bis zum Erreichen des natürlichen Menopausenalters wird sowohl von den ESHRE- als auch von den NICE-Leitlinien empfohlen [1, 2]. Zum Zeitpunkt der Diagnose der POI sollte eine Osteodensitometrie durchgeführt werden. Ein Nutzen routinemäßiger Knochendichtekontrollen unter laufender Therapie ist laut Dachverband für Osteologie nicht belegt [32]. Die ESHRELeitlinie empfiehlt dennoch, die Osteodensitometrie mindestens alle 5 Jahre zu wiederholen, wenn bei der initialen Messung eine Osteoporose vorliegt [1].

Rocca et al., haben eine reduzierte Lebenserwartung bei Frauen mit POI gezeigt [33]. Dies ist vor allem auf das gesteigerte Risiko für kardiovaskuläre Erkrankungen zurückzuführen, das in zahlreichen Studien nachgewiesen wurde. Daher sollten die Betroffenen zusätzliche kardiovaskuläre Risikofaktoren vermeiden. Eine HRT wirkt sich positiv auf Blutdruck, Plasmalipide, Insulinresistenz und Endothelfunktion aus [34-37] und sollte daher empfohlen werden [1]. Eine jährliche Reevaluation des kardiovaskulären Risikos anhand von Blutdruck, BMI und Nikotinabusus sollte ebenfalls erfolgen. Bei Frauen mit Turner-Syndrom sollten zusätzlich Lipide, Nüchternglukose und $\mathrm{HbA}_{1 \mathrm{c}}$ jährlich beurteilt werden, und die Betreuung sollte zusammen mit einem erfahrenen Kardiologen erfolgen.

Die Diagnose POI kann sich zudem negativ auf das psychosexuelle Wohlbefinden auswirken [38]. Deshalb ist es wichtig, die Patientinnen nach Problemen im Bereich der Sexualität zu fra- 
gen. Urogenitale Beschwerden wie vaginale Trockenheit, Dyspareunie, Dysurie, Inkontinenz und gesteigerte Miktionsfrequenz können trotz einer adäquaten systemischen Östrogensubstitution auftreten. In diesem Fall kann zusätzlich lokal Östrogen appliziert werden. Wenn eine HRT kontraindiziert ist, kann die Verwendung von Gleitgel hilfreich sein. Die transdermale Applikation von Testosteron bei Libidoverlust im „off label use“ ist hingegen umstritten [39].

Die Auswirkung des Hormonmangels auf neurologische Funktionen ist unklar, aber vor allem eine iatrogene POI infolge von Adnexektomie scheint mit einer Verschlechterung der verbalen Gedächtnisfunktion, beschleunigtem kognitivem Abbau und erhöhtem Risiko für Demenz und Parkinson assoziiert zu sein $[40,41]$.

\section{Hormonersatztherapie}

Eine Hormonersatztherapie bei POI ist nicht nur zur Behandlung der Östrogenentzugssymptomatik, sondern auch zur Primärprävention von kardiovaskulären Erkrankungen und in der Osteoprotektion dringend indiziert. Eine Substitution mit $17 \beta$-Östradiol ist gegenüber Ethinylöstradiol tendenziell zu bevorzugen, da der osteoprotektive Effekt von Ethinylöstradiol geringer zu sein scheint. $\mathrm{Ob}$ ein kombiniertes orales Kontrazeptivum (KOK) ebenfalls eine ausreichende Osteoprotektion garantiert, ist derzeit noch unklar. Laut einer 2012 publizierten Metaanalyse hemmen KOK bei perimenopausalen Frauen den Knochenabbau [42]. Für das Kollektiv der Frauen mit POI gibt es allerdings kaum Daten. Eine unverblindete randomisierte Studie mit 59 POI-Patientinnen verzeichnete unter HRT einen leichten Anstieg der BMD im Zeitraum von 2 Jahren, aber auch unter KOK blieb die Knochendichte zumindest stabil [43]. Zwei randomisierte Trials zeigten bei POI-Patientinnen unter Östradiol außerdem niedrigere Blutdruckwerte im Vergleich zu Ethinylöstradiol $[34,44]$. Es könnte folglich ein positiver Effekt auf das kardiovaskuläre Risiko der HRT im Vergleich zu KOK bestehen. Wenn gleichzeitig Kontrazeptionsbedarf besteht, kann aber angesichts der geringen Unterschiede dennoch ein kombiniertes orales Kontrazeptivum eingesetzt werden [1]. Es gibt keine Studien zum Einsatz von konjugiertem equinem Östrogen oder den neuen KOK mit Östradiol bei POI-Patientinnen.

Die transdermale Applikation von Östradiol umgeht im Gegensatz zur oralen Applikation den First-Pass-Effekt in der Leber, sodass bei einer geringeren Dosierung ausreichende Plasmalevel erreicht werden können [45]. Außerdem sind die metabolischen und thrombogenen Effekte geringer [46]. Der Zielplasmawert von $50 \mathrm{bis} 100 \mathrm{pg} / \mathrm{ml}$ kann transdermal mit einer Dosis von $100 \mu \mathrm{g}$ Östradiol täglich erreicht werden [47]. Bei einer oralen
Anwendung sind 2-4 mg täglich nötig [48].

Bei intaktem Uterus muss die HRT zwingend durch ein Gestagen ergänzt werden. In Bezug auf die Endometriumprotektion sind die verschiedenen Gestagene wahrscheinlich gleich effizient [49], wobei die Evidenz bei synthetischen Gestagenen höher ist. Natürliches mikronisiertes Progesteron scheint ein günstigeres kardiovaskuläres Risikoprofil zu haben $[48,50]$ und sich weniger auf das Brustkrebsrisiko auszuwirken [51]. Die Gestagenapplikation kann kontinuierlich oder zyklisch erfolgen. Die kontinuierliche Gabe scheint eine geringfügig bessere
Hier steht eine Anzeige.

\section{Springer}


Endometriumprotektion zu gewährleisten [52]. Die zyklische Gabe sollte mindestens alle 12 Wochen für 14 Tage erfolgen. In einem kontinuierlichen Regime ist eine Dosis von $1 \mathrm{mg}$ Norethisteron oder $2 \mathrm{mg}$ MPA täglich ausreichend; sequenziell sollten $10 \mathrm{mg}$ MPA täglich für jeweils 10-14 Tage verabreicht werden. Alternativ können $200 \mathrm{mg}$ mikronisiertes Progesteron oral oder vaginal gegeben werden [52]. Bei Kontrazeptionsbedarf kommt auch ein levonorgestrelhaltiges IUD infrage [53].

Die Präferenz der Patientin sollte bei der Verschreibung einer HRT unbedingt berücksichtigt werden, um die Compliance zu gewährleisten.

Die Hormonersatztherapie sollte bis zum Erreichen des natürlichen Menopausenalters von circa 50 Jahren fortgesetzt werden.

\section{Fazit für die Praxis}

- Bei einer Amenorrhö länger als 4 Monate vor dem 40. Lebensjahr sollte eine hormonelle Abklärung erfolgen.

- Bei zweimaligen FSH-Werten über $25 \mathrm{IU} / \mathrm{ml}$ liegt eine $\mathrm{POI}$ vor.

- Bei POI soll eine genetische Abklärung erfolgen.

- Eine autoimmune Ursache sollte ausgeschlossen werden.

- Es sollte eine initiale Osteodensitometrie durchgeführt werden.

- Vor einer gonadotoxischen Therapie müssen Patientinnen über die Möglichkeiten einer Fertilitätsprotektion aufgeklärt werden.

- Eine Schwangerschaft sollte je nach Anamnese in einem Zentrum betreut werden.

- Eine HRT sollte zur Primärprävention von kardiovaskulären Erkrankungen und zur Osteoprotektion bis zum Alter von 50 erfolgen.

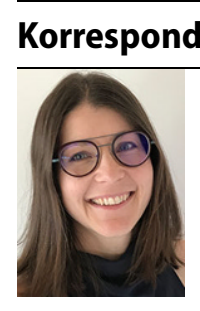

Universitätsklinik

für Gynäkologische

Endokrinologie und

Reproduktionsmedizin,

Medizinische Universität Anichstraße 35, 6020 Innsbruck, Österreich katharina.feil@tirolkliniken.at

\section{Prof. Dr. Bettina Toth}

Universitätsklinik für Gynäkologische Endokrinologie und Reproduktionsmedizin, Medizinische Universität

Anichstraße 35, 6020 Innsbruck, Österreich

bettina.toth@i-med.ac.at

Funding. Open access funding provided by University of Innsbruck and Medical University of Innsbruck.

\section{Einhaltung ethischer Richtlinien}

Interessenkonflikt. K. Feil, A.L. Zippl und B. Toth geben an, dass kein Interessenkonflikt besteht.

Für diesen Beitrag wurden von den Autoren keine Studien an Menschen oder Tieren durchgeführt. Für die aufgeführten Studien gelten die jeweils dort angegebenen ethischen Richtlinien.

Open Access. Dieser Artikel wird unter der Creative Commons Namensnennung 4.0 International Lizenz (http://creativecommons.org/licenses/by/4.0/deed de) veröffentlicht, welche die Nutzung, Vervielfältigung, Bearbeitung, Verbreitung und Wiedergabe in jeglichem Medium und Format erlaubt, sofern Sie den/die ursprünglichen Autor(en) und die Quelle ordnungsgemäßnennen, einen Linkzur Creative Commons Lizenz beifügen und angeben, ob Änderungen vorgenommen wurden.

\section{Literatur}

1. Webber L et al (2016) ESHRE Guideline: Management of women with premature ovarian insufficiency. Hum Reprod 31(5):926-937

2. Lumsden MA et al (2016) Diagnosis and management of menopause: The National Institute of Health and Care Excellence (NICE) guideline. JAMA Intern Med 176(8):1205-1206

3. Stuenkel CA et al (2015) Treatment of symptoms of the menopause: An endocrine society clinical practice guideline. J Clin Endocrinol Metab 100(11):3975-4011

4. Coulam CB, Adamson SC, Annegers JF (1986) Incidence of premature ovarian failure. Obstet Gynecol 67(4):604-606

5. Chemaitilly W et al (2017) Premature ovarian insufficiency in childhood cancer survivors: A report from the St. Jude lifetime cohort. J Clin Endocrinol Metab 102(7):2242-2250

6. Mishra GD et al (2017) Early menarche, nulliparity and the risk for premature and early natural menopause. Hum Reprod 32(3):679-686
7. Cade JE etal (2017) Cohort profile: The UKwomen's cohort study (UKWCS). Int JEpidemiol 46(2):e11

8. AWMF (2018) Peri- and postmenopause diagnosis and interventions. Guideline of the German Society of Gynocology and Obstetrics (S3 Level, AWMF Registry No 015-062)

9. Goldenberg RL et al (1973) Gonadotropins in women with amenorrhea. The use of plasma follicle-stimulating hormone to differentiate women with and without ovarian follicles. Am J Obstet Gynecol 116(7):1003-1012

10. La Marca A et al (2009) Primary ovarian insufficiency due to steroidogenic cell autoimmunity is associated with a preserved pool of functioning follicles.JClinEndocrinol Metab94(10):3816-3823

11. Pasquino AM et al (1997) Spontaneous pubertal development in Turner's syndrome. Italian Study Group for Turner's Syndrome. J Clin Endocrinol Metab 82(6):1810-1813

12. Gravholt CHetal (2017) Clinical practice guidelines for the care of girls and women with Turner syndrome: Proceedings from the 2016 Cincinnati International Turner Syndrome Meeting. Eur J Endocrinol 177(3):G1-G70

13. Hagerman RJ et al (2017) Fragile X syndrome. Nat Rev Dis Primers 3:17065

14. Sherman SL (2000) Premature ovarian failure in the fragileX syndrome.Am JMed Genet 97(3):189-194

15. Brabant $\mathrm{G}$ et al (2002) Autoimmun polyglanduläre Syndrome: Aspekte zu Pathogenese, Prognose und Therapie. Dtsch Arztebl Int 99(21):1428

16. Förster $G$ et al (1999) Polyglandular autoimmune syndrome type II: Epidemiology and forms of manifestation. Dtsch Med Wochenschr 124(49):1476-1481

17. Ahonen $P$ et al (1990) Clinical variation of autoimmune polyendocrinopathy-candidiasisectodermal dystrophy (APECED) in a series of 68 patients. NEngl J Med 322(26):1829-1836

18. Szlendak-Sauer K et al (2016) Autoimmune polyglandular syndrome type 3 (APS-3) among patients with premature ovarian insufficiency (POI). Eur J Obstet Gynecol Reprod Biol 203:61-65

19. Dittrich R et al (2018) Fertility preservation for patients with malignant disease. Guideline of the DGGG, DGU and DGRM (S2k level, AWMF Registry No. 015/082, November 2017)—Recommendations and statements for girls and women. Geburtshilfe Frauenheilkd 78(6):567-584

20. Wallace WH, Thomson AB, Kelsey TW (2003) The radiosensitivity of the human oocyte. Hum Reprod 18(1):117-121

21. Wallace WH et al (2005) Predicting age of ovarian failure after radiation to a field that includes the ovaries. Int J Radiat Oncol Biol Phys 62(3):738-744

22. Coccia ME et al (2011) Ovarian surgery for bilateral endometriomas influences age at menopause. Hum Reprod 26(11):3000-3007

23. Bachelot A et al (2009) Phenotyping and genetic studies of 357 consecutive patients presenting with premature ovarian failure. Eur J Endocrinol 161(1):179-187

24. van Kasteren YM, Schoemaker J (1999) Premature ovarian failure: A systematic review on therapeutic interventions to restore ovarian function and achieve pregnancy. Hum Reprod Update 5(5):483-492

25. Beckmann MW et al (2019) Concept paper on the technique of cryopreservation, removal and transplantation of ovarian tissue for fertility preservation. Geburtshilfe Frauenheilkd 79(1):53-62

26. Jensen AK et al (2017) Cryopreservation of ovarian tissue for fertility preservation in a large cohort of 
young girls: Focus on pubertal development. Hum Reprod 32(1):154-164

27. Schleedoorn MJ et al (2019) To freeze or not to freeze? An update on fertility preservation in females with turner syndrome. Pediatr Endocrinol Rev 16(3):369-382

28. Wallace WH et al (2013) Long term follow-up of survivors of childhood cancer: Summary of updated SIGN guidance. BMJ 346:f1190

29. Hudson MM (2010) Reproductive outcomes for survivors of childhood cancer. Obstet Gynecol 116(5):1171-1183

30. Jeve YB et al (2016) Donor oocyte conception and pregnancy complications: A systematic review and meta-analysis. BJOG 123(9):1471-1480

31. Popat VB et al (2009) Bone mineral density in estrogen-deficient young women. J Clin Endocrinol Metab 94(7):2277-2283

32. Thomasius F et al (2018) DVO Leitlinie 2017 zur Prophylaxe, Diagnostik und Therapie der Osteoporose bei postmenopausalen Frauen und Männern: Kurzversion Bd. 27, S154-160

33. Rocca WA et al (2006) Survival patterns after oophorectomy in premenopausal women: A population-based cohort study. Lancet Oncol 7(10):821-828

34. Langrish JP et al (2009) Cardiovascular effects of physiological and standard sex steroid replacement regimens in premature ovarian failure. Hypertension 53(5):805-811

35. Darling GM et al (1997) Estrogen and progestin compared with simvastatin for hypercholesterolemia in postmenopausal women. $\mathrm{N}$ Engl J Med 337(9):595-601

36. Sumino $\mathrm{H}$ et al (2003) Hormone replacement therapy decreases insulin resistance and lipid metabolism in Japanese postmenopausal women with impaired and normal glucose tolerance. Horm Res 60(3):134-142

37. Kalantaridou SN et al (2004) Impaired endothelial function in young women with premature ovarian failure: Normalization with hormone therapy.JClin Endocrinol Metab 89(8):3907-3913

38. Liao KL, Wood N, Conway GS (2000) Premature menopause and psychological well-being JPsychosom Obstet Gynaecol 21(3):167-174

39. Elraiyah T et al (2014) Clinical review: The benefits and harms of systemic testosterone therapy in postmenopausal women with normal adrenal function: A systematic review and meta-analysis. JClin Endocrinol Metab 99(10):3543-3550

40. Rocca WA, Grossardt BR, Shuster LT (2014) Oophorectomy, estrogen, and dementia: A 2014 update. Mol Cell Endocrinol 389(1-2):7-12

41. Rocca WA et al (2008) Increased risk of parkinsonism in women who underwent oophorectomy before menopause. Neurology 70(3):200-209

42. Nappi $\mathrm{C}$ et al (2012) Hormonal contraception and bone metabolism: A systematic review. Contraception 86(6):606-621

43. Cartwright B et al (2016) Hormone replacement therapy versus the combined oral contraceptive pill in premature ovarian failure: A randomized controlled trial of the effects on bone mineral density.J Clin Endocrinol Metab 101(9):3497-3505

44. Guttmann H et al (2001) Choosing an oestrogen replacement therapy in young adult women with Turner syndrome. Clin Endocrinol (Oxf) 54(2):159-164

45. Goodman MP (2012) Are all estrogens created equal? A review of oral vs. transdermal therapy. JWomens Health (Larchmt) 21(2):161-169

46. Renoux C, Dell'Aniello S, Suissa S (2010) Hormone replacement therapy and the risk of venous thromboembolism: A population-based study. JThromb Haemost 8(5):979-986

47. Popat VB et al (2008) Normalization of serum luteinizing hormone levels in women with 46,XX spontaneous primary ovarian insufficiency. Fertil Steril 89(2):429-433

48. Steingold KA et al (1991) Comparison of transdermal to oral estradiol administration on hormonal and hepatic parameters in women with premature ovarian failure. J Clin Endocrinol Metab 73(2):275-280

49. The Writing Group for the PEPI Trial (1996) Effects of hormone replacement therapy on endometrial histology in postmenopausal women. The Postmenopausal Estrogen/Progestin Interventions (PEPI) Trial.JAMA.

50. Mueck AO (2012) Postmenopausal hormone replacement therapy and cardiovascular disease: The value of transdermal estradiol and micronized progesterone. Climacteric 15(Suppl 1):11-17

51. Fournier A, Berrino F, Clavel-Chapelon F (2008) Unequal risks for breast cancer associated with different hormone replacement therapies: Results from the E3N cohort study. Breast Cancer Res Treat 107(1):103-111

52. Furness $S$ et al (2012) Hormone therapy in postmenopausal women and risk of endometrial hyperplasia.Cochrane Database Syst Rev 8:CD402

53. Ewies AA, Alfhaily F (2012) Use of levonorgestrelreleasing intrauterine system in the prevention and treatment of endometrial hyperplasia. Obstet Gynecol Surv67(11):726-733

Hinweis des Verlags. Der Verlag bleibt in Hinblick auf geografische Zuordnungen und Gebietsbezeichnungen in veröffentlichten Karten und Institutsadressen neutral.
Corinna A. Schön, Katja Wolf

Medizinische Akutversorgung nach sexualisierter Gewalt

Ein forensisch-klinischer Praxisleitfaden

Berlin: Springer 2019, 1., 272 S. 252 Abb., (ISBN: 978-3-662-561737), 44,99 EUR

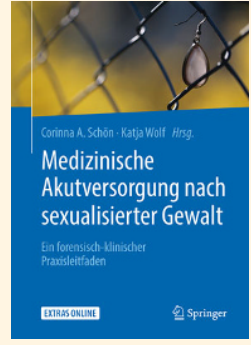

Das Buch gibt in 11 Kapiteln einen umfassenden Überblick über das Thema. Dies ist schon daran ersichtlich, dass die Autorinnen mit Definitionen rechtlichen Aspekten etc. anfangen, den Untersuchungen die Anatomie der Anogenitalregion voranstellen. Dies ist mit Blick auf Unsicherheiten, die in Befundbeschreibungen immer wieder deutlich werden, hilfreich. Es schließen sich die Untersuchungen, einschließlich der Untersuchungsbedingungen und des Umgangs mit den Betroffenen an. Es werden Aspekte der Dokumentation ebenso behandelt wie die Verletzungen, sexuell übertragbare Erkrankungen und Langzeitfolgen. Auch Differentialdiagnosen finden Berücksichtigung. Zahlreiche Checklisten finden sich als Anhang. Die Kapitel sind kleinteilig gegliedert, was auch eine Nutzung als "Nachschlagewerk" für den gerade benötigten Aspekt ermöglicht. Die bekannten Gestaltungsregeln eines Springer-Buches sind ebenfalls hilfreich, die zahlreichen Fotos von exzellenter Qualität. Ich werde dieses Buch gerade auch gynäkologisch tätigen Kolleginnen und Kollegen empfehlen. Für Neulinge in einer der mit dem Thema befassten Fachdisziplin ist es ebenfalls ein guter Einstieg. In einer Institutsbibliothek sollte es als aktuelles Übersichtswerk allen zugänglich sein.

S. Banaschak (Köln) 